Synthetic division was not used this time because it was felt that the round-off error would be carried into the reduced polynomial. Observing the erratic property of the last digits it was concluded that the limit of accuracy in the worst case is about 12 decimal places. There seems to be no question for values of $m$ from 1 to 5 where $n=0,1$, and 2 .

A check of the $b_{j}$ was made through the fact that

$$
\sum_{j=1}^{m} b_{j}=\int_{0}^{1} x^{n} d x=\frac{1}{n+1}
$$

for each polynomial of degree $m$ associated with $x^{n}$. Here again the limit of accuracy is put at about the twelfth decimal place for the worst possible cases (i.e., with $m$ and $n$ both large). If one wishes to round off values to ten or eleven decimal places there should be no doubt of the accuracy.

I wish to acknowledge the helpful suggestions of the referee concerning the form of the first three paragraphs. The calculations were carried out in the Numerical Analysis Laboratory of the University of Wisconsin on the IBM Type 650. I am grateful for the use of the 18-digit floating decimal routine of Eugene Albright and his helpful suggestions in programming. The calculations and work were supported by funds of the Wisconsin Alumni Research Foundation granted by the Graduate Research Committee.

University of Wisconsin

Herbert Fishman

Madison, Wisconsin

1. R. Courant \& D. Hilbert, Methods of Mathematical Physics, First English edition, Translated and Revised from the German Original, Interscience Publishers, Inc., New York, v. 1, 1953, p. 90-91.

2. P. C. Hammer, O. J. Marlowe, \& A. H. Stroud, "Numerical integration over simplexes and cones," $M T A C$, v. 10, 1956, p. 130-137.

3. A. ERDELYI (editor), Higher Transcendental Functions, v. 2, McGraw-Hill Book Co., Inc., New York, 1953, p. 168-170.

4. William VernoN LovitT, Linear Integral Equations, McGraw'-Hill Book Co., Inc., New York, 1924.

\title{
Tables of the Exponential Integral $\mathrm{Ei}(\mathbf{x})$
}

In some molecular structure calculations it is desirable to have values of the integral $\mathrm{Ei}(x)$ to higher accuracy than is provided by the standard tables [1]. Direct computation of the values needed is extremely tedious over a wide range of the argument intermediate between the regions where expansions about zero and infinity are useful. However, if a net of sufficiently accurate values of $\mathrm{Ei}(x)$ has already been computed, then the generation of additional values intermediate between these becomes a problem of a considerably lesser magnitude. A few such values have been published $[2,3]$. Kotani $[4]$ has given a considerable number of values in his most valuable compilation, but unfortunately they are of somewhat fewer significant figures than under discussion here. A prohibitive amount of labor would be required to make such an extensive accurate table that only simple interpolation formulas need be used. However, a most useful intermediate objective could be achieved if a table were prepared with entries sufficiently closely 
spaced that rapidly convergent series expansions could be used to obtain further values. Accordingly, an 18-significant digit table was prepared by the means described below.

The two usual ways of obtaining values of $\operatorname{Ei}(x) a b$ initio are from the Taylor series

$$
\mathrm{Ei}(x)=\gamma+\ln |x|+x+\frac{x^{2}}{2 \cdot 2 !}+\frac{x^{3}}{3 \cdot 3 !}+\cdots
$$

and from the asymptotic formula

$$
\operatorname{Ei}(x) \sim e^{x}\left[\frac{1}{x}+\frac{1}{x^{2}}+\frac{2 !}{x^{3}}+\frac{3 !}{x^{4}}+\cdots\right] .
$$

The Taylor series becomes inconvenient beyond about $|x|=4$, and the asymptotic formula is incapable of giving sufficiently accurate values for $|x|<50$. It is necessary to generate values in the range $4<|x|<50$ with the aid of a suitable recursion formula.

Two recursion formulas have been employed recently for this purpose. The first, used by Ruedenberg, Roothaan, and Jaunzemis [5], may be written in the form

$$
\mathrm{Ei}(x)=\mathrm{Ei}(x-h)+\sum_{k=1}^{\infty} \frac{(-h)^{k}}{k !} A_{k-1}(-x)
$$

where $A_{n}(x)$ is defined as

$$
A_{n}(x)=x^{-n-1} e^{-x} n ! \sum_{k=0}^{n} \frac{x^{k}}{k !}
$$

The integrals $A_{n}(x)$ have been tabulated by Kotani [4] for positive $x$, though not all to sufficient accuracy for use in (3). $A_{n}(x)$ for negative $x$ can be obtained, also not with sufficient accuracy for use in (3) from Kotani's tables of $B_{n}(x)$ and the relation $B_{n}(x)=(-1)^{n+1} A_{n}(-x)-A_{n}(x)$.

A second recursion formula for $\operatorname{Ei}(x)$, used by Kotani [4], may be cast in the form

$$
e^{-x} \operatorname{Ei}(x)=e^{-h}\left[e^{-(x-h)} \operatorname{Ei}(x-h)\right]+x^{-1} \sum_{k=0}^{\infty} x^{-k} R_{k}(h)
$$

where the coefficients $R_{n}(h)$ are related to the $A_{n}$ by

$$
R_{n}(h)=n !-h^{n+1} A_{n}(h) .
$$

This second expansion is far more convenient than the first, as very little computation is required to apply it for given $h$ to different values of $x$. Moreover, the coefficients $R_{n}(h)$ may be obtained without great labor by consideration of the recursion relation between $R_{n}(h)$ and $R_{n-1}(h)$,

$$
R_{n}(h)=n R_{n-1}(h)-h^{n} e^{-h}, \quad n \geq 1,
$$


together with the value $R_{0}(h)=1-e^{-h}$. Equation (7) may be verified by induction. A third recursion formula, intermediate in character between (3) and (5), was given by Gram [3].

Equations (5) and (7) were employed to compute the values of $e^{-x} \operatorname{Ei}(x)$ presented here for $|x| \geq 4$. By using the formula to proceed from smaller to larger $x$, no loss in significant figures accompanies repeated use of the formula, and the final accuracy is determined by the number of figures carried in the $R_{n}$. Starting values of $\operatorname{Ei}(-50)$ and $\operatorname{Ei}(4)$ were computed by (2) and (1), respectively, and the values of $\mathrm{Ei}(-4)$ and $\mathrm{Ei}(50)$ obtained by repeated use of the recursion formulas were checked against values computed, respectively: from (1) and (2). Twenty-one significant figures were carried throughout the calculations, and the final results were rounded off to 18 . Some intermediate values were also obtained in more than one way as a check. The same work sheets were used to compute for both positive and negative values of $x$, thus further reducing the possibility of error in formulation of the $x^{-k} R_{k}$. All check conditions were satisfied to 20 or more significant digits.

The 21-figure values of $e^{-x} \operatorname{Ei}(x)$ were multiplied by 19-figure values of $e^{x}$ [6] to obtain the values of $\operatorname{Ei}(x)$ presented to 18 figures. Table 1 gives our final values of $\operatorname{Ei}(-x)$ and $-e^{x} \operatorname{Ei}(-x)$, together with additional values obtained by Bretschneider [2]. In table 2 are presented the corresponding quantities for positive $x$. Table 3 gives values of the $R_{n}(h)$ to sufficient precision to interpolate from the nearest table value anywhere for $|x|>4$. Miscellaneous constants used are listed in table 4.

\section{TABLE 1. Exponential Integrals, Negative Arguments}

The numbers in parentheses are the powers of 10 by which the entries so marked must be multiplied.

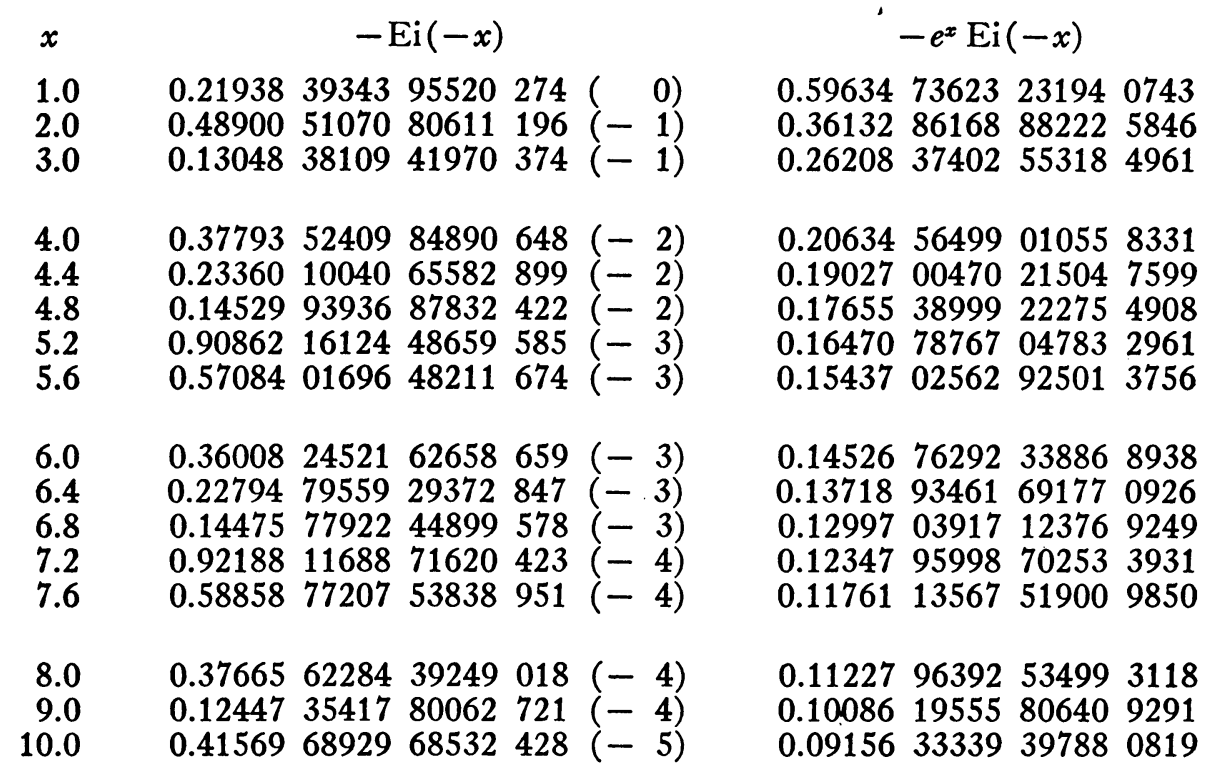


TABLE OF THE EXPONENTIAL INTEGRAL EI $(x)$

TABLE 1-Continued

$x$

11.0

12.0

13.0

14.0

15.0

16.0

17.0

18.0

19.0

20.0

21.0

22.0

23.0

24.0

25.0

26.0

27.0

28.0

29.0

30.0

31.0

32.0

33.0

34.0

35.0

36.0

37.0

38.0

39.0

40.0

41.0

42.0

43.0

44.0

45.0

46.0

47.0

48.0

49.0

50.0

$$
-\operatorname{Ei}(-x)
$$

0.140030030424744178 (- 5)

$0.475108182467249393(-6)$

$0.162186621880143287(-6)$

$0.556563111114518212(-7)$

$0.191862789214786698(-7)$

$0.664048724944104278(-8)$

$0.230643198982165449(-8)$

$0.803609034482867766(-9)$

$0.280782909706079527 \quad(-9)$

$0.983552529064988169(-10)$

$0.345320126714675627(-10)$

$0.121493789562043727(-10)$

$0.428268479566567262(-11)$

$0.151230589399970577(-11)$

$0.534889975534021664(-12)$

$0.189468588567497824(-12)$ $0.672063743526204038(-13)$

$0.238694151193373316(-13)$

$0.848775977835356284(-14)$

$0.302155201068881254(-14)$

$0.107676703861623826(-14)$ $0.384096180122506683(-15)$ $0.137138434844874657 \quad(-15)$ $0.490067611839278771(-16)$ $0.175270593899473720(-16)$

$0.627333900976224159(-17)$ $0.22470 \quad 2069758857 \quad 122 \quad(-17)$

$0.805410691429074987(-18)$

$\begin{array}{lllll}0.28887 & 79301 & 52270 & 100 & (-18)\end{array}$

$0.103677326145165697(-18)$

$0.372316677645997772(-19)$

$0.133779088100117751 \quad(-19)$

$0.480949655695001785(-20)$

$0.172995987428164776(-20)$

$0.622569080946238364(-21)$

$0.224153175974429975(-21)$

$0.807419784272581395 \quad(-22)$

$0.29096 \quad 6419040584 \quad 234 \quad(-22)$

$0.104898116423680237(-22)$

$0.378326402955045902(-23)$

$$
-e^{x} \operatorname{Ei}(-x)
$$

0.0838417788603459505

0.0773261331389192306

$0.0717535335 \quad 244623954$

0.0669325181834396292

$\begin{array}{lllll}0.06272 & 02791 & 07409 & 2418\end{array}$

0.0590081036085564362

$0.055711755743476 \quad 7274$

0.0527649444026250680

0.0501147797954799196

0.0477185454959608417

0.0455413616545051706 0.0435544646926178827

$0.04173 \quad 39215 \quad 560006238$

$\begin{array}{lllll}0.04005 & 96555 & 23840 & 3225\end{array}$

$\begin{array}{llll}0.03851 & 46988 & 44904 & 0221\end{array}$

$\begin{array}{lllll}0.03708 & 46128 & 39349 & 6946\end{array}$ $\begin{array}{lllll}0.03575 & 70332 & 31537 & 5283\end{array}$

$\begin{array}{lllll}0.03452 & 13102 & 36847 & 3608\end{array}$

$\begin{array}{lllll}0.03336 & 82211225610743\end{array}$

$\begin{array}{llll}0.03228 & 97387 & 58980 & 1252\end{array}$

$\begin{array}{lllll}0.03127 & 88438 & 29256 & 7742\end{array}$ $\begin{array}{llll}0.03032 & 93713 & 77333 & 2475\end{array}$ 0.0294358845813415053 0.0285935702760013970 0.0277981519719529646

$0.02704 \quad 58170 \quad 44635 \quad 3756$ $0.02633 \quad 31554 \quad 69674 \quad 7075$ $\begin{array}{lllll}0.02565 & 71080 & 22614 & 4181\end{array}$ $\begin{array}{llll}0.02501 & 49222 & 79330 & 0217\end{array}$ 0.0244041150796285763

0.0238224403724518529 0.0232678615631998260 0.0227385276442614456 $\begin{array}{lllll}0.02223 & 27525 & 18155 & 0389\end{array}$ 0.0217489970257852704

$\begin{array}{lllll}0.02128 & 58532 & 75605 & 0273\end{array}$ $\begin{array}{lllll}0.02084 & 20309 & 37076 & 1867\end{array}$ 0.0204163452169651554 0.0200077062821898425 0.0196151099301148704 
TABLE 2. Exponential Integrals, Positive Arguments

The numbers in parentheses are the powers of 10 by which the entries so marked must be multiplied.

\begin{tabular}{|c|c|c|c|c|c|c|c|c|}
\hline$x$ & & & $i(x)$ & & & $e^{-x} \mathrm{Ei}$ & $(x)$ & \\
\hline $\begin{array}{l}1.0 \\
2.0 \\
3.0\end{array}$ & $\begin{array}{l}0.18951 \\
0.49542 \\
0.99338\end{array}$ & $\begin{array}{l}17816 \\
34356 \\
32570\end{array}$ & $\begin{array}{ll}35593 & 676 \\
00189 & 016 \\
62541 & 656\end{array}$ & $\begin{array}{l}(1) \\
(1) \\
(1)\end{array}$ & $\begin{array}{l}0.69717 \\
0.67048 \\
0.49457\end{array}$ & $\begin{array}{l}48832 \\
27097 \\
64013\end{array}$ & $\begin{array}{l}35066 \\
90073 \\
48641\end{array}$ & $\begin{array}{l}0688 \\
2810 \\
2350\end{array}$ \\
\hline $\begin{array}{l}4.0 \\
4.4 \\
4.8 \\
5.2 \\
5.6\end{array}$ & $\begin{array}{l}0.19630 \\
0.26008 \\
0.34697 \\
0.46624 \\
0.63101\end{array}$ & $\begin{array}{l}87447 \\
97327 \\
88987 \\
85050 \\
78597\end{array}$ & $\begin{array}{ll}00562 & 200 \\
16051 & 460 \\
37753 & 276 \\
57967 & 478 \\
42992 & 615\end{array}$ & $\begin{array}{l}(2) \\
(2) \\
(2) \\
(2) \\
(2)\end{array}$ & $\begin{array}{l}0.35955 \\
0.31932 \\
0.28555 \\
0.25720 \\
0.23334\end{array}$ & $\begin{array}{l}20078 \\
10053 \\
48567 \\
89914 \\
18047\end{array}$ & $\begin{array}{l}63620 \\
85318 \\
95924 \\
23568 \\
99632\end{array}$ & $\begin{array}{l}6962 \\
4003 \\
4435 \\
2427 \\
7448\end{array}$ \\
\hline $\begin{array}{l}6.0 \\
6.4 \\
6.8 \\
7.2 \\
7.6\end{array}$ & $\begin{array}{l}0.85989 \\
0.11793 \\
0.16270 \\
0.22568 \\
0.31457\end{array}$ & $\begin{array}{l}76214 \\
48657 \\
70875 \\
78077 \\
18784\end{array}$ & $\begin{array}{ll}24392 & 048 \\
00181 & 887 \\
71431 & 415 \\
01063 & 810 \\
98083 & 578\end{array}$ & $\begin{array}{l}(2) \\
(3) \\
(3) \\
(3) \\
(3)\end{array}$ & $\begin{array}{l}0.21314 \\
0.19595 \\
0.18121 \\
0.16849 \\
0.15742\end{array}$ & $\begin{array}{l}73100 \\
55338 \\
91105 \\
53143 \\
79475\end{array}$ & $\begin{array}{l}81593 \\
64928 \\
15268 \\
53259 \\
14471\end{array}$ & $\begin{array}{l}6031 \\
3941 \\
3455 \\
2881 \\
5487\end{array}$ \\
\hline $\begin{array}{r}8.0 \\
9.0 \\
10.0\end{array}$ & $\begin{array}{l}0.44037 \\
0.10378 \\
0.24922\end{array}$ & $\begin{array}{l}98995 \\
78290 \\
28976\end{array}$ & $\begin{array}{ll}34838 & 269 \\
71708 & 959 \\
24187 & 776\end{array}$ & $\begin{array}{l}(3) \\
(4) \\
(4)\end{array}$ & $\begin{array}{l}0.14773 \\
0.12808 \\
0.11314\end{array}$ & $\begin{array}{l}09983 \\
43565 \\
70204\end{array}$ & $\begin{array}{l}73400 \\
23213 \\
73410\end{array}$ & $\begin{array}{l}9966 \\
8681 \\
7780\end{array}$ \\
\hline $\begin{array}{l}11.0 \\
12.0 \\
13.0 \\
14.0 \\
15.0\end{array}$ & $\begin{array}{l}0.60714 \\
0.14959 \\
0.37197 \\
0.93192 \\
0.23495\end{array}$ & $\begin{array}{l}06374 \\
53266 \\
68849 \\
51363 \\
58524\end{array}$ & $\begin{array}{ll}09861 & 151 \\
63975 & 289 \\
06890 & 356 \\
39653 & 713 \\
90768 & 304\end{array}$ & $\begin{array}{l}(4) \\
(5) \\
(5) \\
(5) \\
(6)\end{array}$ & $\begin{array}{l}0.10140 \\
0.09191 \\
0.08407 \\
0.07749 \\
0.07187\end{array}$ & $\begin{array}{l}28126 \\
45454 \\
90291 \\
22514 \\
35404\end{array}$ & $\begin{array}{l}36185 \\
08896 \\
67225 \\
92093 \\
92410\end{array}$ & $\begin{array}{l}3129 \\
5894 \\
1376 \\
0115 \\
7085\end{array}$ \\
\hline $\begin{array}{l}16.0 \\
17.0 \\
18.0 \\
19.0 \\
20.0\end{array}$ & $\begin{array}{l}0.59556 \\
0.15166 \\
0.38779 \\
0.99509 \\
0.25615\end{array}$ & $\begin{array}{l}09986 \\
37894 \\
04330 \\
07251 \\
65266\end{array}$ & $\begin{array}{ll}70837 & 002 \\
04251 & 688 \\
59744 & 350 \\
04684 & 476 \\
40565 & 888\end{array}$ & $\begin{array}{l}(6) \\
(7) \\
(7) \\
(7) \\
(8)\end{array}$ & $\begin{array}{l}0.06702 \\
0.06278 \\
0.05906 \\
0.05575 \\
0.05279\end{array}$ & $\begin{array}{l}15610 \\
78642 \\
04044 \\
29076 \\
77952\end{array}$ & $\begin{array}{l}41399 \\
32855 \\
06932 \\
96429 \\
79648\end{array}$ & $\begin{array}{l}0873 \\
1665 \\
4489 \\
0258 \\
1322\end{array}$ \\
\hline $\begin{array}{l}21.0 \\
22.0 \\
23.0 \\
24.0 \\
25.0\end{array}$ & $\begin{array}{l}0.66127 \\
0.17114 \\
0.44396 \\
0.11541 \\
0.30059\end{array}$ & $\begin{array}{l}18635 \\
46713 \\
63698 \\
15391 \\
50906\end{array}$ & $\begin{array}{ll}54849 & 213 \\
00363 & 668 \\
30271 & 221 \\
84918 & 295 \\
52554 & 869\end{array}$ & $\begin{array}{r}(8) \\
(9) \\
(9) \\
(10) \\
(10)\end{array}$ & $\begin{array}{l}0.05014 \\
0.04774 \\
0.04555 \\
0.04356 \\
0.04174\end{array}$ & $\begin{array}{l}13386 \\
02599 \\
92944 \\
94088 \\
64774\end{array}$ & $\begin{array}{l}46825 \\
85690 \\
77218 \\
38540 \\
50664\end{array}$ & $\begin{array}{l}6145 \\
4452 \\
8886 \\
5760 \\
5301\end{array}$ \\
\hline $\begin{array}{l}26.0 \\
27.0 \\
28.0 \\
29.0 \\
30.0\end{array}$ & $\begin{array}{l}0.78429 \\
0.20496 \\
0.53645 \\
0.14059 \\
0.36897\end{array}$ & $\begin{array}{l}40991 \\
49711 \\
11859 \\
91957 \\
32094\end{array}$ & $\begin{array}{ll}89818 & 637 \\
98808 & 124 \\
23146 & 942 \\
58406 & 905 \\
07274 & 197\end{array}$ & $\begin{array}{l}(10) \\
(11) \\
(11) \\
(12) \\
(12)\end{array}$ & $\begin{array}{l}0.04007 \\
0.03852 \\
0.03709 \\
0.03576 \\
0.03452\end{array}$ & $\begin{array}{l}02837 \\
37569 \\
23813 \\
37344 \\
71217\end{array}$ & $\begin{array}{l}69455 \\
74926 \\
73152 \\
29948 \\
92361\end{array}$ & $\begin{array}{l}1129 \\
3125 \\
0594 \\
2813 \\
8461\end{array}$ \\
\hline $\begin{array}{l}31.0 \\
32.0 \\
33.0 \\
34.0 \\
35.0\end{array}$ & $\begin{array}{l}0.96945 \\
0.25500 \\
0.67146 \\
0.17698 \\
0.46690\end{array}$ & $\begin{array}{l}55759 \\
43566 \\
40184 \\
03724 \\
55014\end{array}$ & 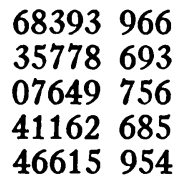 & $\begin{array}{l}(12) \\
(13) \\
(13) \\
(14) \\
(14)\end{array}$ & $\begin{array}{l}0.03337 \\
0.03229 \\
0.03128 \\
0.03033 \\
0.02943\end{array}$ & $\begin{array}{l}32862 \\
41738 \\
27441 \\
28152 \\
89370\end{array}$ & $\begin{array}{l}79497 \\
81757 \\
22948 \\
54435 \\
25758\end{array}$ & $\begin{array}{l}7246 \\
5328 \\
5202 \\
4435 \\
9265\end{array}$ \\
\hline
\end{tabular}


TABLE 2-Continued

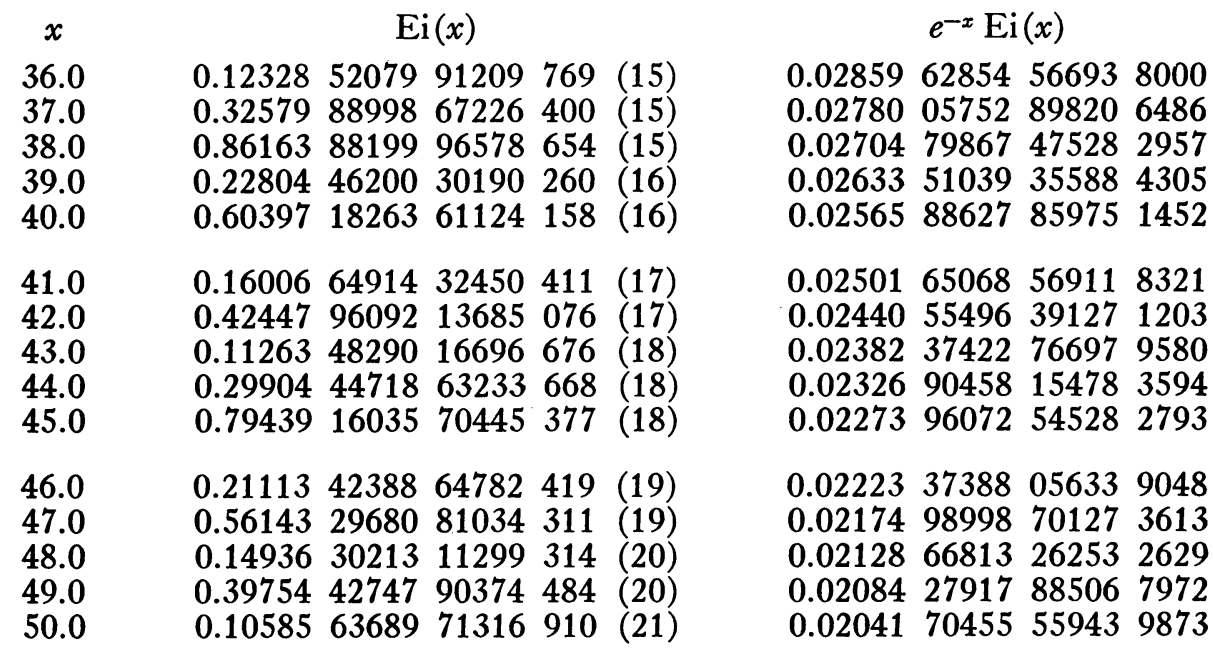

TABLE 3. Interpolation Coefficients

\begin{tabular}{|c|c|}
\hline$n$ & $R_{n}(1)$ \\
\hline $\begin{array}{l}1 \\
2 \\
3 \\
4\end{array}$ & $\begin{array}{llll}0.63212 & 05588 & 28557 & 67840 \\
0.26424 & 11176 & 57115 & 35681 \\
0.16060 & 27941 & 42788 & 3920 \\
0.11392 & 89412 & 56922 & 854 \\
0.08783 & 63238 & 56249 & 10\end{array}$ \\
\hline $\begin{array}{l}5 \\
6 \\
7 \\
8\end{array}$ & $\begin{array}{llll}0.07130 & 21781 & 09803 & 2 \\
0.05993 & 36274 & 87377 \\
0.05165 & 59512 & 4019 \\
0.04536 & 81687 & 501 \\
0.04043 & 40775 & 80\end{array}$ \\
\hline 1 & $\begin{array}{ll}0.03646 & 13346 \\
0.03319 & 52397 \\
0.03046 & 3435 \\
0.02814 & 522 \\
0.02615 & 36\end{array}$ \\
\hline & $\begin{array}{l}0.024424 \\
0.02291 \\
0.0216 \\
0.020 \\
0.02\end{array}$ \\
\hline
\end{tabular}

\begin{tabular}{llll}
\multicolumn{4}{c}{$R_{n}(0.5)$} \\
0.39346 & 93402 & 87366 & 57640 \\
0.09020 & 40104 & 31049 & 86459 \\
0.02877 & 53559 & 33941 & 3733 \\
0.01050 & 97353 & 37744 & 942 \\
0.00413 & 07751 & 18940 & 18
\end{tabular}

0.0016997924786811

$0.00072 \quad 1713314077$

0.00031347241953

0.0001385189668

0.000062040506

0.00002808996

0.00001283204

0.0000059057

0.000002735

0.00000127

$$
R_{n}(0.4)
$$

0.32967995396436069926 0.06155193555010497896 $0.0158526637 \quad 345076698$ $\begin{array}{lllll}0.00465 & 75082 & 57242 & 094\end{array}$ 0.00146983985045601

0.0000006

\subsection{1 0.000165100976689 0.00005745447340 0.0000203348418 0.000007293198}

0.00000264383

0.00000096686 0.0000003562 0.000000132 0.00000005

$$
R_{n}(0.3)
$$

$$
R_{n}(0.2)
$$

$$
R_{n}(0.1)
$$

0.18126924692201814133 0.01752309630642176960 0.0022969624897242648 $0.0369363131 \quad 1376677411$ 0.0071989863661789403 $\begin{array}{lllll}0.00159 & 48671 & 40130438\end{array}$ 0.00037884097299984 $0.00034 \quad 10414 \quad 445489397$ 0.000054196573270988

\subsection{6}

0.000024043049579

0.00000628440219

0.0000016701340

0.000000449681

0.00000898902536998

0.00000153538402291

$0.00000 \quad 02679345210$

0.0000000475254402

0.000000008538816

0.09516258196404042684 0.00467884016044446952 0.00030930614052934331 0.00002308100355207035 0.0000018402724046854

0.00000000155013 0.00000000028382 0.0000000000523 0.00000003358 0.0000000093

0.0000000000097

$\begin{array}{llll}0.00000 & 01529 & 87843 \quad 068\end{array}$ $\begin{array}{lllll}0.00000 & 00130 & 89640 & 37\end{array}$ 0.0000000011437408 0.0000000001015521 0.000000000009131

0.00000000000083 0.00000000000008 
TABLE 3-Continued

\begin{tabular}{llll} 
& \multicolumn{2}{c}{$R_{n}(0.05)$} \\
0.04877 & 05754 & 99285 & 99091 \\
0.00120 & 91042 & 74250 & 29045 \\
0.00004 & 01349 & 87248 & 79589 \\
0.00000 & 15012 & 83683 & 7984 \\
0.00000 & 00599 & 50832 & 064 \\
& & & \\
0.00000 & 00024 & 94965 & 16 \\
0.00000 & 00001 & 06831 & 23 \\
0.00000 & 00000 & 04670 & 6 \\
0.00000 & 00000 & 00207 & 5 \\
0.00000 & 00000 & 00009 &
\end{tabular}$$
R_{n}(0.02)
$$

0.01980132669324469778 $0.0001973532 \quad 2710959173$ 0.0000026269848964813 0.0000000393653029900 0.000000000629424231

0.00000000001048540 0.00000000000017969 0.0000000000000031 0.0000000000000001

$$
R_{n}(0.04)
$$

0.03921056084767679056 0.00077898328158386218 0.00002070346052400723 0.0000006198574662730 0.000000019808900862

\subsection{4 0.00000000002260090 0.0000000000007906 0.0000000000000281 0.000000000000001}

$$
\begin{array}{llll}
\multicolumn{4}{c}{R_{n}(0.01)} \\
0.00995 & 01662 & 50831 & 94643 \\
0.00004 & 96679 & 13340 & 26589 \\
0.00000 & 03308 & 43305 & 6150 \\
0.00000 & 00024 & 80083 & 0958 \\
0.00000 & 00000 & 19834 & 046
\end{array}
$$

0.00000000000016524 0.00000000000000142

$$
R_{n}(0.03)
$$

0.02955446645149182307 $0.00044 \quad 110044503657776$ 0.00000879990987949816 $\begin{array}{lllll}0.00000 & 0197700232 & 6848\end{array}$ 0.000000004740048565

0.00000000011841636 0.00000000000304336 0.0000000000000799 0.0000000000000021

TABLE 4. Constants

$$
\begin{aligned}
& \gamma=0.577215664901532860606512 \quad \text { (Euler's constant) } \\
& \log _{10} e=0.434294481903251827651129
\end{aligned}
$$

$h$

0.01

0.02

0.03

0.04

0.05

0.10

0.20

0.30

0.40

0.50

1.00

$$
e^{h}
$$

$\begin{array}{lllll}1.01005 & 01670 & 84168 & 05754 & 21655 \\ 1.02020 & 13400 & 26755 & 81016 & 01439 \\ 1.03045 & 45339 & 53516 & 85561 & 24400 \\ 1.04081 & 07741 & 92388 & 22675 & 70448 \\ 1.05127 & 10963 & 76024 & 03969 & 75176 \\ 1.10517 & 09180 & 75647 & 62481 & 17078 \\ 1.22140 & 27581 & 60169 & 83392 & 10720 \\ 1.34985 & 88075 & 76003 & 10398 & 37443 \\ 1.49182 & 46976 & 41270 & 31782 & 48530 \\ 1.64872 & 12707 & 00128 & 14684 & 86508 \\ 2.71828 & 18284 & 59045 & 23536 & 02875\end{array}$

$$
e^{-h}
$$

$\begin{array}{lllll}0.99004 & 98337 & 49168 & 05357 & 39060\end{array}$ $\begin{array}{lllll}0.98019 & 86733 & 06755 & 30222 & 08141\end{array}$ 0.9704455335485081769325284 $0.960789439152323 \quad 2094392107$ $\begin{array}{llllll}0.95122 & 94245 & 00714 & 00909 & 14253\end{array}$ $0.90483 \quad 74180359595731642491$ 0.8187307530779818586699355 $0.74081822068171786606 \quad 68738$ $\begin{array}{lllllll}0.67032 & 00460 & 35639 & 30074 & 44329\end{array}$ $\begin{array}{llllll}0.60653 & 06597 & 12633 & 42360 & 37995\end{array}$ 0.3678794411714423215955238

Our tables agree entirely with the values Bretschneider [2] and Gram [3] give for integers from -10 to 20 . They differ from Kotani's table [4] by 1 in his last place for a number of values of the argument, and by larger amounts for the following values of $x: 6.0,8.0,9.0,10.0,12.0,-18.0,-24.0,-32.0,-33.0$, $-43.0,-48.0$.

Harvard University

Frank E. HARRIS

Cambridge, Massachusetts

1. NYMTP (A. N. LowAN, technical director), Tables of Sine, Cosine and Exponential Integrals, v. 1, NBS, 1940, p. 31 ; Circular and Hyperbolic Functions, Exponential and Sine and Cosine Integrals, Factorial Function and Allied Functions, Hermitian Probability Functions, BAAS Mathematical Tables, v. 1, second edition, 1946, p. viii and 31-33.

2. C. A. BRetschneIDER, "Über die abgeleiteken Vierecke usw.," Archiv der Math. u. Phys., first series, v. 3,1843 , p. 27-34.

3. J. P. GRAM, "Unders $\varnothing$ gelser angaaende Maengden af Primtal under en given Graense," Danske Vidensk. Selsk. Skr., 6 Raekke, naturvid. og math. Afd., v. 2, 1884, p. 184-288. 
4. M. Kotani, A. Amemiya, E. Ishiguro, \& T. Kimura, Table of Molecular Integrals, Maruzen Co., btd., Tokyo, 1955.

5. K. Ruedenberg, C. C. J. RoothaAn, \& W. Jaunzemis, "Study of two-center integrals useful in calculations of molecular structure. III. A unified treatment of the hybrid, Coulomb, and one-electron integrals," $J$. Chem. Phys., v. 24, 1956, p. 201-20.

6. NBS, Applied Mathematics Series, No. 14, Tables of the Exponential Function $e^{x}$, U. S. Gov. Printing Office, Washington, D. C., 1951.

7. G. Placzer, "The Functions $E_{n}(x)=\int_{1}^{\infty} e^{-x u} u^{-n} d u$," with Appendices by G. Blanch and the MTP, NBS Applied Mathematics Series, No. 37, p. 57-111.

\section{On the Computation of $\log Z$ and $\operatorname{arc} \tan Z$}

In a previous note, Clenshaw [1] has given numerical values of coefficients for the expansion of some transcendental functions in Chebyshev polynomials. In particular, he tabulates the coefficients for $\log (1+x)$ and $\operatorname{arc} \tan x$ to nine decimal places. Here, treating these functions in a more general form, we determine precise theoretical coefficients and show that the development leads to formulas for computation in the complex domain.

The Chebyshev polynomials of the first kind which we use in the range $-1 \leq x \leq 1$ are defined as

$$
T_{n}(x)=\cos n \theta, \quad x=\cos \theta
$$

For a discussion of the properties of these functions, see the work of Lanczos [2]. If $f(x)$ is bounded and continuous in a given range, then the expansion

$$
f(x)=\frac{1}{2} C_{0}+\sum_{k=1}^{\infty} C_{k} T_{k}(x)
$$

is convergent, and

$$
C_{k}=\frac{2}{\pi} \int_{-1}^{1} \frac{f(x) T_{k}(x) d x}{\sqrt{1-x^{2}}}
$$

The transformation $x=2 y-1$ shifts the range to $0 \leq y \leq 1$, and we denote the "shifted" polynomial as $B_{n}(y)$.

Consider $f(y)=1 /(y+a)$. Utilizing the above together with a known formula [3], we find

$$
\begin{gathered}
1 /(y+a)=\left[1+2 \sum_{k=1}^{\infty}(-)^{k} q^{k} B_{k}(y)\right] /\left(a^{2}+a\right)^{\frac{1}{2}} ; q=2 a+1-2\left(a^{2}+a\right)^{\frac{1}{2}} \\
0 \leq y \leq 1 ; \quad|\arg a| \leq \pi / 2, \quad a \neq 0 .
\end{gathered}
$$

From a numerical point of view, (4) is pathologic. If $a=1$, the Taylor series development of $1 /(y+1)$ is slowly convergent near $y=1$; at $y=1$ it is divergent. However, (4) is precise and rapidly convergent in this region. The above is a striking example to show the strength of a Chebyshev expansion and the comparative weakness of a Taylor series expansion. The integral of (4) is not patho- 